\section{Paper Electrophoresis of a Purified Specific Antibody}

RECENTLY, several workers ${ }^{1}$ have tried to fractionate gamma-globulins and immune sera by electrophoresis. Such experiments have only given the mean properties of all gamma-globulin proteins, since non-antibody as well as antibody proteins occur in immune sera. We have now carried out paper electrophoresis of a purified specific antibody.

Antigenic azo-globulin was prepared by isolating pseudoglobulins from beef serum using ammonium sulphate ${ }^{2}$, and coupling with diazotized anthranilic acid. Four rabbits were immunized as previously described $^{3}$, and, when a high antibody titre was established, these were killed by exsanguination and antibody precipitated from the antiserum with homologous antigen. The antibody was separated from the complex using sodium chloride solution and $0 \cdot 1 N$ hydrochloric acid, as previously described ${ }^{4}$, and the antibody estimated by micro-Kjeldahl nitro. gen determination. Paper electrophoresis was earried out with a Jouan Paris, model 1603, apparatus and No. 204 papers measuring $400 \mathrm{~mm}$. $\times 27.5 \mathrm{~mm}$. Alkaline conditions ( $300 \mathrm{~V} ., 100$ m.amp., $6 \mathrm{hr}$., with a veronal-sodium veronal buffer, $p \mathrm{H} 8.8$ ) and acid conditions ( $200 \mathrm{~V} ., 100 \mathrm{~m} . \mathrm{amp} ., 5 \mathrm{hr}$., with a sodium veronal-acetate buffer, $p H$ 4) were both used ${ }^{5}$. In each experiment we ran one strip with $0.1 \mathrm{ml}$. normal human serum as a control, and two strips with $0.05 \mathrm{ml}$. of antibody solution $(825 \mathrm{mgm}$. protein $/ 100 \mathrm{ml}$.). After electrophoresis, the strips were dried at $90^{\circ}$, stained for $15 \mathrm{~min}$. in a bromphenol blue solution $(0.2 \mathrm{gm}$. dye and $65 \mathrm{gm}$. mercuric chloride in $200 \mathrm{ml} .96$ per cent ethanol), washed in tap water and dried at $100^{\circ}$. Colour intensity was measured with an optical densitometer. Pure antibody showed a single homogeneous peak at both acid and alkaline $p \mathrm{H}$, corresponding at alkaline $p \mathrm{H}$ to the peak of the serum gamma-globulin.

\section{Shevket Tekman}

AYTEN UGUR

Department of Biological Chemistry,

University of Istanbul,

Turkey.

Nov. 30 .

${ }^{1}$ Cann, J. R., Brown, R. A., and Kirkwood, J. G., J. Amer. Chem. Soc., 71, 2687 (1949); J. Jiol. Chem., 181, I61 (1949). Chem.
J. R., Brown, R. A., Kirkwood, J. G., and Hink, J. H., J. Biol. Chem., 185, 663 (1950). Cann, J. R., Campbell, D, H., Brown, R. A., and Kirkwood, J. G., J. Amer. Chem. Soc., '73, 4611 (1951) Jankovic, B. D., and Krijnen, H. W., Nature, 171, 982 (1953) 2 Haurowitz, F., Saraftan, K., and Schwerin, P., J. Immunol., 40,
391 (1941).

${ }^{3}$ Haurowitz, F., J. Immunol., 43, 331 (1942).

' Haurowitz, F., and Tekman, S., Nature, 157, 335 (1946). Haurowitz, F., Teknan, S., Bilen, M., and Schwerin, P., Biochem. J.,

'Mich relis, L., Biochem. Z., 234, 139 (1931).

\section{Neuromuscular Block caused by Acetylcholine}

$I_{T}$ has long been known that large doses of acetylcholine may cause a neuromuscular block ${ }^{1}$. This block has been thought to be due to the ability of acetylcholine to depolarize persistently the end-plate regions of the muscle fibres ${ }^{2}$. No direct studies on the relation of such a neuromuscular block to the musele membrane potential have, however, been carried out.

In the present investigation this relationship has been studied on the sartorius nerve - muscle propara-

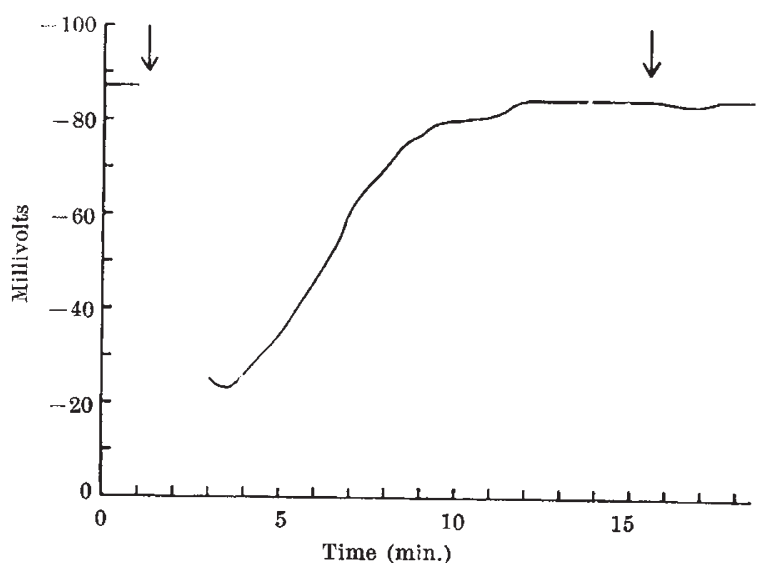

Fig. 1. Continuous registration of the membrane potcntial at the end-plate region of a single muscle flbre. At the arrows, acetylcholine iodide was added to the muscle bath, giving a concentration of $10 \times 10^{-8}$ and $20 \times 10^{-6} M$

tion of the frog (Rana temporaria). The membrane potentials and the end-plate potentials of single muscle fibres were recorded with capillary microelectrodes of external tip diameter less than $0 \cdot 5 \mu$ filled with $3 M$ potassium chloride (for details of technique see ref. 3 ).

In the presence of $1 \times 10^{-6} M$ neostigmine bromide, acetylcholine iodide (Hoffmann-La Roche) in a concentration of $5-20 \times 10^{-\beta} M$ caused a neuromuscular. block. When the initial fibrillary twitches of the muscle fibres had subsided (about 60 sec.), the tip of the microelectrode was re-inserted at the end-plate region of a single muscle fibre and the membrane potential continuously recorded. These concentrations of acetylcholine caused a considerable reduction of the membrane potential at the end-plate region. Without removal of the acetylcholine the depolariza. tion, however, spontaneously subsided and the membrane potential was restored to its original value within 7-15 min. as shown in Fig. 1. A subsequent addition of acetylcholine to the bath did not significantly change the membrane potential.

During the period of depolarization, a neuromuscular block developed and persisted despite repolarization of the membrane to the normal level. End-plate potentials could sometimes be recorded during the phase of depolarization, but never in the later phase when the membrane potential again was normal. The neuromuscular transmission remained completely blocked so long as acetylcholine was in the muscle bath and could only be restored slowly by washing the muscle in Ringer's fluid for $15-45 \mathrm{~min}$. During the period of washing, the membrane potential remained normal and, preceding the relief of block, end-plate potentials again appeared. These end-plate potentials were markedly increased in size by repetitive stimulation of the motor nerve, and eventually an action potential could be elicited (Fig. 2).

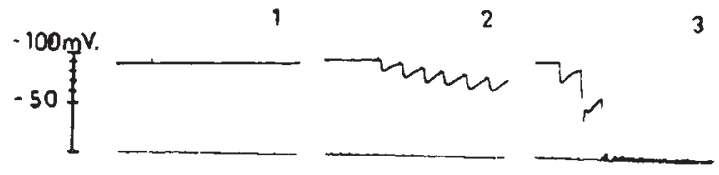

Fig. 2. (1) End-plate potentials have disappeared during the later phase of the neuromuscular block caused by $10 \times 10^{-6} M$ acetyl end-plate potentials are recorded which are increased in's fluid. repetitive stimulation of the motor nerve increased in size by to an action potential. (3) Time. $5 \mathrm{~m}$.sec. 